\title{
Review on digitalization of basic routine procedures in histopathology laboratories
}

\begin{abstract}
Slowly but surely, healthcare is going digital. Many of the recent innovations in healthcare, from telemedicine and smart devices to the growing capabilities in managing big data, can be traced back to the adoption of new digital technologies that have fostered a different way of working. Across medicine's varied specialty areas, however, the adoption and progress of digital technology advances varies significantly. Digitalization in histopathology laboratory in Nigeria, is still a big setback, but there are digitalization in some of the usual routine practices like the automatic tissue processor which has overtaken the usual manual processor. In the area of sectioning, staining of tissues, mounting of slide and slide reading, we still face major setback in that regard. An improvement in digital diagnostic work, will go a long way in improving basic histopathological practice. From the reported usage of digital tools we can also conclude that possibilities for an overview image, measurements and annotations are much appreciated parts of the digital environment..
\end{abstract}

Keywords: digitalization, histopathology, basic
Volume 3 Issue 5 - 2017

\author{
Nosa Terry Omorodion, ${ }^{1,2}$ Peter Uwadiegwu \\ Achukwu, ${ }^{2}$ Godfrey Innocent lyare, 3,4 \\ Ajanwachuku Ogbonna Wilson' \\ 'Department of health Services, University of Benin, Nigeria \\ ${ }^{2}$ Department of Medical Laboratory Science, University of \\ Nigeria, Nigeria \\ ${ }^{3}$ Department of Medical Laboratory Science, University of \\ Benin, Nigeria \\ ${ }^{4}$ Department of Medical Laboratory Science, Igbinedion \\ University, Nigeria
}

\begin{abstract}
Correspondence: Nosa Terry Omorodion, Health Services Department, University of Benin, Benin City, Edo state, Nigeria, Tel 08136742270,Email terry.omorodion@uniben.edu
\end{abstract}

Received: December 08, 2017 | Published: December 29, 2017

\section{Introduction}

Digitalization is the process of converting of analog to digital. Slowly but surely, healthcare is going digital. Many of the recent innovations in healthcare, from telemedicine and smart devices to the growing capabilities in managing big data, can be traced back to the adoption of new digital technologies that have fostered a different way of working. Across medicine's varied specialty areas, however, the adoption and progress of digital technology advances varies significantly. Digitalization in histopathology laboratory in Nigeria, is still a big setback, but there are digitalization in some of the usual routine practices like the automatic tissue processor which has overtaken the usual manual processor. In the area of sectioning, staining of tissues, mounting of slide and slide reading, we still face major setback in that regard. Digitalization in routine histopathology laboratory also offers new possibilities not available within the conventional microscope. A promising technique is automatic scoring and other kinds of digital image analysis (DIA). In a review, ${ }^{1}$ conclude that current DIA methods are able to produce quantitative assessments of immunohistochemically stained slides with a similar variability as manual assessment. In other studies, quantitative digitalization methods have been shown to outperform manual work for certain applications, such as in Ki67 proliferation assessment ${ }^{2}$ and prediction of recurrence in prostate cancer. ${ }^{3}$ However, ${ }^{3}$ highlight the fact that a full-scale investigation of DIA methods considering all aspects of the clinical situation is still lacking. In histopathology, and particularly in molecular pathology, there are an increasing number of tests and an increasing demand to support faster, more-accurate disease diagnosisand subsequent treatment and care-particularly for cancer (Figure 1).

Histopathology laboratories are among the last healthcare environments to benefit from fully digitized workflows. In most hospitals and pathology laboratories today, tissue-based diagnoses are still rendered by pathologists using the traditional process of analyzing glass slide samples using a microscope. This traditional way of working comes with challenges-aside from the inherent delays built into its processes-including the time and risks associated with transferring physical glass slides. However, change is starting to emerge, with the introduction and adoption of digital histopathology systems in both research and clinical settings. Many building blocks for implementing digital pathology are available today. Progress has clearly been made in recent years in terms of resolution, image quality, and throughput of scanners; whereas, storage costs have been continuously declining. Therefore, many pathology labs are now facing the question if digital pathology is mature enough to be considered for large-scale implementation in routine clinical practice.

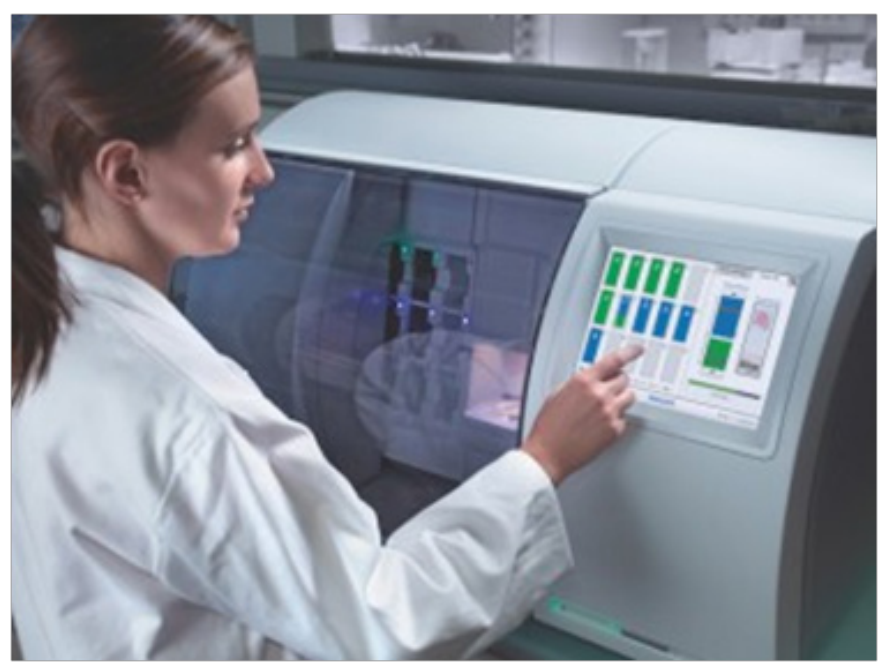

Figure I A lab technician initiates scanning with the IntelliSite ultra fast scanner.

\section{Advantages of digitalization in histopathology}

Personalized medicine and genomics offer the promise of individualizing healthcare-a particularly strong motivator in the field of oncology, where the demand for better and more-efficient care 
permeates the dialogue of healthcare professionals. Histopathology will play a central role in this evolution. As the work of diagnosing and treating cancer becomes more complex, however, so too does the work of the pathologist and, by extension, that of the entire pathology department. While clinicians in the specialty of histopathology may be decreasing, oncologists are facing mounting pressure to curtail the pervasive disease at the center of their specialty. Statistics compiled by the International Agency for Research on Cancer project that new cancer cases will rise $70 \%$ over the next 2 decades, from 14 million in 2012 to 22 million per year in $2035 .{ }^{4}$ Even more troubling is the lack of concordance in the definitive diagnoses of cancer. A study from the American Society of Clinical Oncology found that for common HER2 breast cancer, approximately $20 \%$ of testing may be inaccurate-and this finding is for just one type of cancer. ${ }^{5}$ Another report issued by the UK's National Patient Safety Agency found that protracted decision making in pathology accounts for $41 \%$ of delays in cancer diagnosis, and is a leading cause of patient safety incidents. ${ }^{6}$ Histopathologists are still just beginning to learn how to manage the massive amounts of rich imaging data they procure every day, but it is clear that there is room for improvement over existing processes. Now is the time when pathologists most need flexibility in harnessing the imaging data at their disposal, in order to put the process of definitive diagnosis on a path toward making personalized medicine a widespread reality ${ }^{7}$ (Figure 2).

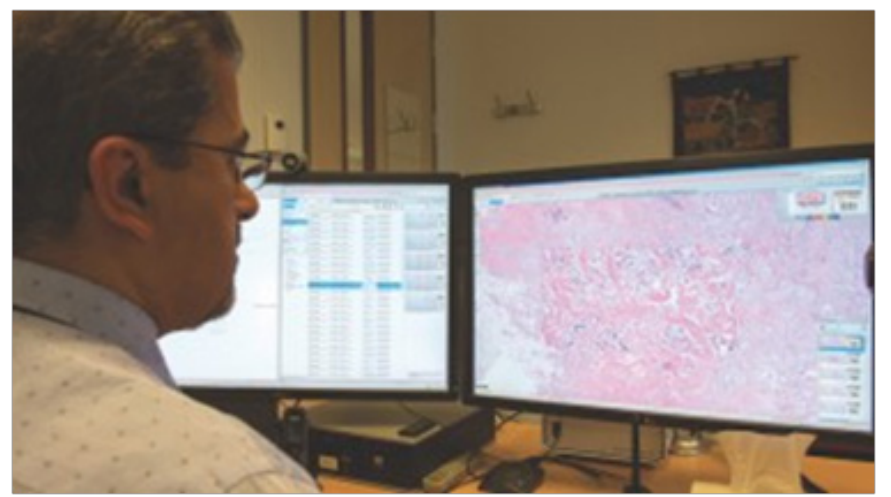

Figure $\mathbf{2}$ The case viewer of the IntelliSite pathologist suite.

The transition from microscope-based image analysis to digital pathology may seem like a natural progression, in line with the rising tide of widely varied digital technologies that are already being integrated into the practice of medicine. Additional obstacles arise from regulatory concerns, based in part on the difficulty of using traditional approaches in clinical studies to test or reproduce the skill of the microscope-aided human eye and compare it to the power of digital technologies. Consequently, the reproducibility and standardization associated with real-world use of digital pathology technologies have yet to be evaluated on a large scale. Changes of any sort can be difficult, and often involve trial and some time to make adjustments. But experts remain certain that the inevitable digitization of histopathology will lead to numerous benefits and new opportunities for pathology departments, patients, and the future potential of precision oncology.

\section{Digital pathology supports collaboration and efficiency}

As more pathology departments move from traditional to digital workflows, many aspects of histopathologists' daily work are affected. Coincidently, but not surprisingly, such changes mirror those that occurred in radiology departments more than 2 decades ago, when advanced digital imaging technologies were first introduced into clinical practice. Digital pathology promises a similar revolution for the clinical laboratory community, enabling labs to further advance the systematic management of pathology imaging data through improved data management, backup, storage, and archiving capabilities. Two important functions of digital pathology are to search and share histopathological images and reports very quickly. By supplementing workflows with digital pathology solutions, progressive addition with other hospital information systems-such as electronic medical records, laboratory info management systems, and so on-can benefit histopathologists by enabling them to search for patient pathology and related scientific information using more inherent means, such as patient IDs or case IDs

\section{Advancing technologies}

In traditional pathology workflows, glass slide samples are prepared manually, examined under a microscope, and physically shipped for external consultation (Figure 3). This process is timeconsuming and can be precarious, as there is a risk of misplacing or damaging the specimens in transit. By contrast, the latest and most promising imaging modality to disrupt the clinical lab sector is digital pathology whole-slide imaging, which entails the scanning of glass slides to produce precise digital image replicas in turn speeding and simplifying access to histopathology information across the organization. Contributing to the advance of this field is the IntelliSite pathology solution by Philips, Best, the Netherlands, an automated system for creating and managing digital images of pathology specimens. The IntelliSite pathology solution is a highly scalable platform featuring three core elements:

i. IntelliSite ultra fast scanner, a high-throughput bright-field slide scanner designed to accommodate current histopathology needs for routine use in high-volume labs and integrated pathology networks. The scanner has a storage capacity of 300 barcoded slides, which allows the scanner to run overnight. It also supports a continuous scan mode; it can be loaded and reloaded at any time without disrupting the scanning process.

ii. IntelliSite image management system works to improve the efficiency and effectiveness of pathology labs. The open and scalable design offers optimal integration with the lab's workflow and IT infrastructure environment.

iii. IntelliSite pathologist suite, designed to enable pathologists to review cases as quickly as possible; provides easy access to information and resources to enable better-informed decisionmaking.

iv. The system's advanced software tools manage the scanning, storage, presentation and sharing of information (Figure 4).

In traditional pathology workflows, many steps in the specimen preparation process are performed manually, making it difficult to keep track of specimens and where they stand in the process. Using a digital histopathology solution permits histopathologists to identify and keep track of where specific specimens stand in the review process, and facilitates the department to determine whether schedules or protocols are adhered to, or why delays may be occurring (Figure 5). Among the benefits of a digital histopathology solution is the fact that digital slide images can be more appropriately and safely distributed for exterior consultation, by being sent directly to the external consultant, or by giving the professional access to view the images from a secluded location. Efficiencies in lab operations 
are another promising mark of digital pathology. Histopathologists have traditionally performed diagnostic readings by viewing glass slides while standing (or sitting) over a microscope. A noticeable but important element of histopathology laboratory digitization is that it basically changes the pathologist's primary workstation, moving the histopathologist from the microscope to a computer workstation. This change can help to ease the physical fatigue and tension placed on pathologists from leaning over a microscope for many hours a day (Figure 6).

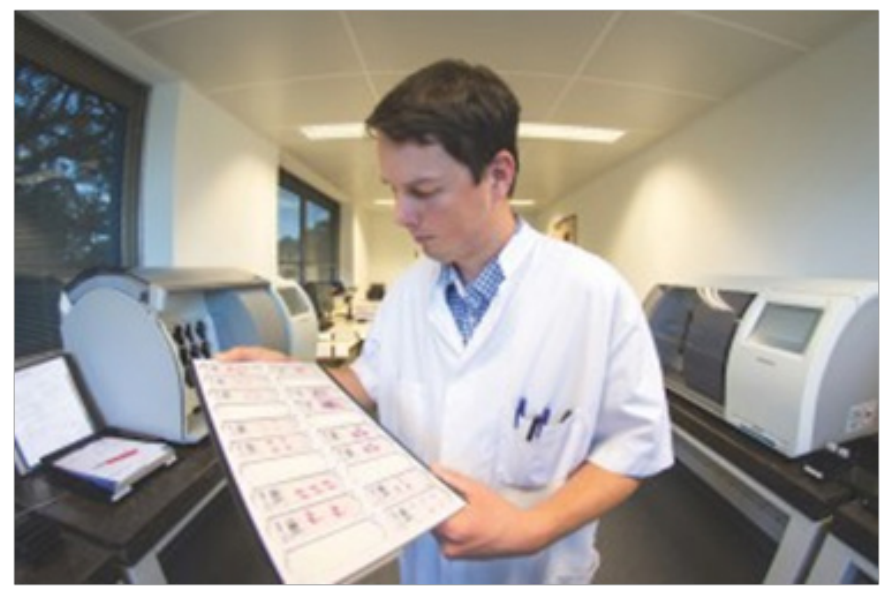

Figure $3 \mathrm{~A}$ lab technician at the Laboratory for Pathology East Netherlands Foundation (LabPON) reviews tissue slides for scanning.

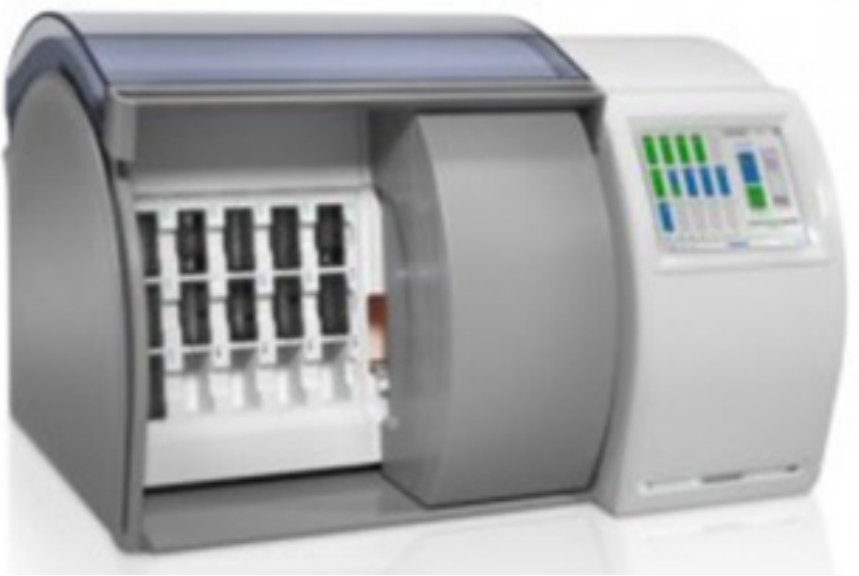

Figure 4The IntelliSite ultra fast scanner by Philips Digital Pathology Solutions, Best, the Netherlands.

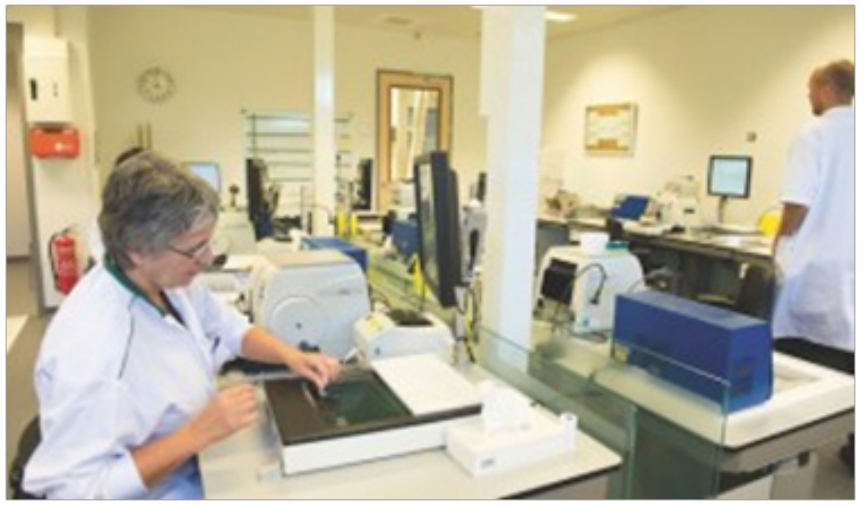

Figure $\mathbf{5}$ In traditional pathology laboratories, many steps in the specimen preparation process are performed manually.

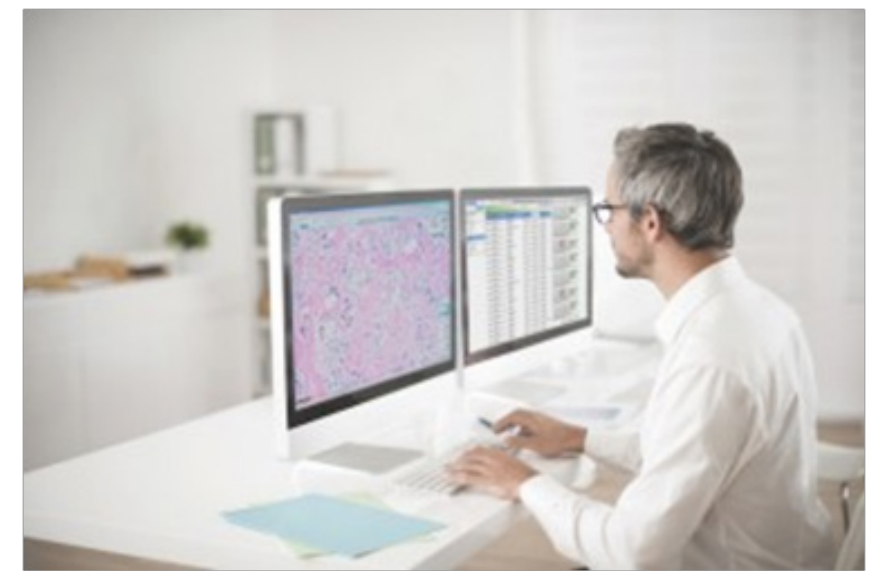

Figure 6 The user screen of the IntelliSite pathologist suite permits side-byside comparison of digital pathology images.

As pathologists adjust to computer-based analysis, and salespersons address opportunities related to the ergonomics of a digital image, the digital workstation offers several advantages when compared to microscopy alone. It is now possible to view different strains of a tissue section side-by-side or in an array format, automatically aligned to allow for a more-direct comparison and, ultimately, outcomes that are more accurate such as in tissue micro array. ${ }^{8}$ Additionally, digital workstations make image measurements easier, and also facilitate the retrieval and comparison of older images from the same patient. As the field of pathology makes its digital transformation, including the use of advanced imaging analytics, it should be underscored that the aim of such transitions is not to replace the knowledge or experience of the pathologist. Instead, digital systems leverage imaging technologies and related computing proficiencies to advance the work and teamwork of histopathologists in and outside of the laboratory. The dream for computational histopathology goes beyond an informatics-centric view, leveraging the core competencies of pathology to understand disease processes at the molecular, individual, and population levels, and the ability of pathologists to effectively incorporate and communicate clinically legal knowledge.

\section{Major setback in implementing digitalization in routine histopathology laboratory}

Major obstacles to converting pathology laboratories to a digital workflow include concerns over the levels of investment required to make the transition, and whether lab revenues can ever be sufficient to produce a reasonable return on such investments. For most laboratory organizations, cost-effectiveness is a key consideration; investments that are not fruitful can frequently become significant factors that inhibit innovation. However, the financial benefits of digital pathology are gradually becoming more evident, as an increasing number of labs are recognizing that digital pathology can increase the revenue of a pathology lab and its hospital system, while also reducing costs through better lab utilization in networked hospital settings-including near elimination of costs for the transport and storage of glass slides. Indeed, as the benefits resulting from digital pathology are shared with other clinical stakeholders, such as oncologists and surgeons, return on investment calculations are beginning to support the transition.

\section{Conclusion}

An improvement in digital diagnostic work will go a long way in improving basic histopathological practice. Digitalization is an 
important tool in improving care delivery. The potential use of wholeslide imaging as the basis for quantitative analysis and the application of tissue micro array and cognitive computing-especially in conjunction with such other data types as next-generation sequencing results-is among a number of recognized drivers leading to the adoption of digital histopathology technologies. Ultimately, digital histopathology will offer new ways to get more information from tissue samples at the cellular and molecular level, and to combine them with large datasets to help translate the promises of 'big data' and 'personalized medicine' into actionable insights. By analyzing, integrating, and presenting the data available from whole-slide imaging within the context of other valuable patient data, pathologists and other researchers can have the tools they need to expand and advance research, increase efficiencies, lower costs, and ultimately improve patient outcomes.

\section{Acknowledgements}

None.

\section{Conflict of interest}

The author declares no conflict of interest.

\section{References}

1. Riber Hansen R, Vainer B, Steiniche T. Digital image analysis: A review of reproducibility, stability and basic requirements for optimal results. APMIS. 2012;120(4):276-289.
2. Gudlaugsson E, Skaland I, Janssen EA, et al. Comparison of the effect of different techniques for measurement of Ki67 proliferation on reproducibility and prognosis prediction accuracy in breast cancer. Histopathology. 2012;61(6):1134-1144.

3. Lee G, Ali S, Veltri R, et al. Cell orientation entropy (COrE): Predicting biochemical recurrence from prostate cancer tissue microarrays. Med Image Comput Comput Assist Interv. 2013;16(3):396-403.

4. Ed Stewart BW, Wild CP Lyon. World cancer report 2014. World health organization, France: Springer; 2014.

5. Wolff AC, Hammond ME, Schwartz JN, et al. American society of clinical oncology/college of American pathologists guideline recommendations for human epidermal growth factor receptor 2 testing in breast cancer. $J$ Clin Oncol. 2007;25(1):118-145.

6. Delayed diagnosis of cancer: thematic review. England: National patient safety agency; 2010.

7. Physician specialty data book. USA: AAMC; 2014.

8. Omorodion NT, Atoigwe BE. Tissue microarray's brief review: an important research tool in molecular pathology. Sokoto journal of medical lab Science. 2016;1(2):80-83. 